\title{
A DATA-DRIVEN APPROACH FOR CREATIVE CONCEPT GENERATION AND EVALUATION
}

\author{
J. Han ${ }^{1, \bigotimes}$, H. Forbes ${ }^{1}$, F. Shi ${ }^{2}$, J. Hao ${ }^{3}$ and D. Schaefer ${ }^{1}$ \\ ${ }^{1}$ University of Liverpool, United Kingdom, ${ }^{2}$ Amazon Web Services, United Kingdom, ${ }^{3}$ Beijing Institute of \\ Technology, China \\ $\triangle$ Ji.Han@liverpool.ac.uk
}

\begin{abstract}
Conceptual design, as an early phase of the design process, is known to have the highest impact on determining the innovation level of design results. Although many tools exist to support designers in conceptual design, additional knowledge, especially knowledge related to emerging technologies, is still often needed. In this paper the authors aim to propose a data-driven creative concept generation and evaluation approach to support designers in incorporating emerging technologies in the new product early development stage. The approach is demonstrated by means of an illustrated example.
\end{abstract}

Keywords: conceptual design, design creativity, evaluation, data-driven design, idea generation

\section{Introduction}

Conceptual design, which involves information gathering, idea generation, and evaluation, is an early stage of the design process. Decisions made in this stage significantly influence aspects of the final product such as performance, reliability, cost, and safety (Hsu and Liu, 2000). Although conceptual design has a high impact on design decisions, better tools are needed to support this process. In addition, the fast adoption and incorporation of emerging technologies into products is one of the main challenges in the context of existing new product design and development. This often requires intensive collaborations of experts from multiple disciplines and backgrounds, which adds to the difficulties of performing conceptual design. Therefore, it is vital to support designers in conceptual design, especially concept generation and evaluation, by providing access to the right tools.

During the design process, $60 \%$ of the design engineers' time is used to explore the needed information and knowledge from unstructured resources (Ullman, 2010). Knowledge is considered a significant role in innovation (Bertola and Teixeira, 2003) and conceptual design, but information gathering is often challenging. Therefore, there is a need to utilise today's vast amounts of data by employing computational techniques to support designers with relevant knowledge in design.

In this paper, the authors aim to propose a computational creative data-driven concept generation and evaluation approach to support designers and design firms in the early phases of the design process. The data-driven approach could facilitate the incorporation of emerging technologies in new product design, and ultimately lead to innovation. An example is included in this study to provide insight on the application of the proposed approach and its feasibility.

In the next section, a review on computational creative concept generation and evaluation is provided. A corresponding new data-driven generation approach for and with evaluation is proposed and 
discussed in section 3. The paper ends with a section on conclusions to be drawn from this work and future research directions.

\section{Related work}

\subsection{Computational concept generation}

Concept or idea generation determines the type of design produced and initiates innovation (Howard et al., 2011). It is one of the most significant but time-consuming stages across the whole design process. An idea is understood as an essential element of thought in visual, abstract or concrete forms (Jonson, 2005), while a concept is the final form of an idea. Generating an idea is fundamental to generating a concept and the generation of ideas has been considered to be the essence of innovation (Sarkar and Chakrabarti, 2011; Cash and Štorga, 2015). However, generating ideas, especially creative ones that are novel, useful and surprising (Han et al., 2019), is challenging.

Idea generation methods and tools are often used to support designers in ideation fluency, flexibility and originality (Childs, 2018). Most of these methods could not generate ideas on their own, instead, these methods lead designers to investigate a larger design space through offering time and structure constraints (Shah et al., 2003). They can help designers produce creative ideas that cannot be generated based on their intuition (Oman et al., 2013). There exist various idea generation methods such as conventional methods including brainstorming and TRIZ, and advanced ones such as bio-inspired design (Helms et al., 2009; Chakrabarti and Shu, 2010; Fu et al., 2014) and 77 design heuristics (Yilmaz et al., 2016).

In order to support designers in idea generation in a more efficient manner, there is a growing interest in developing computational idea generation support tools in recent years. For example, Han et al. (2018b) explored a computation tool, named the Retriever for prompting creative ideation through constructing ontologies to support reasoning. The tool provides new ontologies of a given idea by selecting different ontology relations, along with providing correspondence image mood boards to prompt creativity. However, the tool currently employs a common-sense database, which is limited in its performance. Chen et al. (2019) have proposed an artificial intelligence based data-driven approach for prompting creative design idea generation. The approach could generate novel synthesized images by using GAN (generative adversarial networks) to stimulate designers' creative minds. However, the current approach could only process simple images, and it is also time-consuming to train the GAN model for producing synthesized images.

These computational idea generation support tools could support designers in creative idea generation. However, many tools fail to provide designers with relevant knowledge, especially the knowledge of emerging technologies, in the design context. Lacking the understanding of emerging technologies, such as their functions and characteristics, could cause potential difficulties for designers to apply these technologies into new products. Therefore, it is necessary to provide designers with the knowledge of these emerging technologies to support them in generating creative ideas incorporating the technologies.

\subsection{Concept evaluation}

Concept evaluation plays a significant role in design, which leads to the selection of the most creative concepts. Evaluating a concept is essentially evaluating the degree of creativity of the concept, of which experts are often employed (Sarkar and Chakrabarti, 2011). Therefore, there is an increasing interest in exploring computational approaches for creativity evaluation.

Brown (2015) proposed a computational framework for evaluating design creativity. The framework evaluates a product by comparing it with descriptions of existing products through a set of aspects suggesting creativity. These aspects could involve novelty, resolution, style and so forth. For example, novelty can be assessed by measuring the frequencies of how many similar designs have been produced, and a lower frequency suggests a better novelty. However, it is still challenging to realize this computational design creativity evaluation framework. Hao et al. (2017) developed a functionbased computational method for design concept evaluation, which employs novelty, feasibility and diversity. These three metrics are computationally defined by two knowledge bases created by using 
word embedding technique and circular convolution based on 500,000 granted patents respectively. An experiment has shown that this method could filter a large amount of design concepts. However, the computational method only focuses on measuring function-related aspects of a design concept. Zhang et al. (2017) presented a quantitative approach, which is based on data-driven performance prediction, to evaluate design alternatives (different designs possessing modified function modules in the same product family) in the early stages of engineering design. Two main processes are involved in this computational method: to quantify expert judgements on weights of performance characteristics, and then to rank design alternatives based on values of performance predictions. However, this method has neglected customer preference while determining the performance characteristic weights, which might affect the success of the product. Also, this method is used to evaluate design alternatives rather than assessing different types of products.

Several computational concept evaluation methods are reviewed. Although using computational evaluation methods could avoid the employment of expert assessors, comparing with concept generation methods or tools, fewer studies have explored the use of computational techniques in concept evaluation. Also, the constraints of existing computational evaluation methods have limited value for existing complex design processes. Brown (2015) indicates that computational creative design systems must 'design for evaluation' and 'design with evaluation'. This suggests that the concepts generated in the conceptual design stage should be capable to be evaluated easily, and the evaluation process should be integrated within the concept generation process.

\section{The data-driven approach}

The fast adoption of new technologies is one of the main challenges in the current new product design and development realm, which has also introduced a higher difficulty in conceptual design. Concept generation and evaluation are important but challenging in the design process. They are the main elements constituting the fuzzy front end which determines the content, tasks, times, and costs of new product development, as well as the success of the new product (Eling et al., 2014).

As illustrated in the previous section, most existing tools have lacked the possibility of including information on new and emerging technologies potentially to be incorporated into the design context. This has hampered designers, especially novices, in producing creative ideas for contemporary and futuristic products. In terms of concept evaluation, most of the non-computational methods require the employment of expert assessors, while few methods have employed computational techniques. Therefore, in order to assist designers in conceptual design, there is a need to propose a data-driven computational approach employing today's abundant technologies and vast amounts of information to support the incorporation of fast-emerging technologies in new product design and development.

In the following sections we present a computational concept generation method and a computational evaluation method providing means of an illustrated example of designing a new navigation application using augmented reality (AR). A data-driven computational concept generation and evaluation approach is then proposed based on these methods, followed by a critical discussion.

\subsection{A computational concept generation method}

In addition to producing creative ideas, designers also need to incorporate new technologies in products to meet people's needs. Understanding how people interact and perceive new technologies is thereby vital in concept generation.

Thereby, there is a need to come up with a computational concept generation method providing relevant emerging technology knowledge. Conducting observations, surveys and interviews could collect the required information, but this could be time-consuming and costly. A computational database containing knowledge of people's perception of various new technologies could help to solve this issue.

Shi et al. (2017) have proposed a knowledge base, named 'B-Link', containing design and engineering information. The knowledge base was created through using text mining and data analytic techniques, such as natural-language-processing, itemset mining and disparity filter. Subsequent probability and velocity network analyses are employed to retrieve design information, which have established a unified standard to quantify the correlation degrees for the relations between directly and indirectly linked nodes. Sarica et al. (2020) have explored another knowledge base 'TechNet', which contains 
over four million engineering terms. TechNet is constructed by employing the latest natural language processing techniques for extracting terms that represent engineering concepts from six million US patent documents. The latest word embedding models were used in TechNet for training vector presentations of the engineering terms contained, which provide a basis for semantic distance or relevance calculation for forming the semantic network or knowledge base. A recent study has demonstrated the use of TechNet to explore design concepts for designing future 'flying cars' (Sarica et al., 2019). Luo et al. (2019) have developed the InnoGPS system which provides the retrieval of design concepts from the patent data in various technology domains. It retrieves terms and concepts at the domain level based on the knowledge distance across different domains, which is different from BLink and TechNet whose retrievals are guided at the term or concept level.

B-Link is used in this study, as it is shown that the B-Link retrieval results are consistent with people's judgements. The B-Link knowledge base is in a 'WordNet' form connecting design and engineering concepts via semantic relations, as shown in Figure 1. The figure has presented an example showing knowledge of an emerging technology 'augmented reality'. In the figure, 'augmented reality' is linked to terms such as 'virtual reality', 'interactive learning environment', 'simulation', 'modelling', and so on, which indicates how 'augmented reality' is associated to other technologies and knowledge. However, the knowledge shown in the figure is only a demonstration of a small proportion of the knowledge that can be retrieved by B-Link. Besides, each knowledge 'node', such as 'virtual reality' and 'simulation', could also lead to further explorations of that knowledge. This knowledge base could support designers in understanding 'augmented reality', and thereby facilitate the incorporation of this technology in new products during the concept generation stage. Thus, the B-Link knowledge base could be applied to provide technology-related knowledge for assisting concept generation.

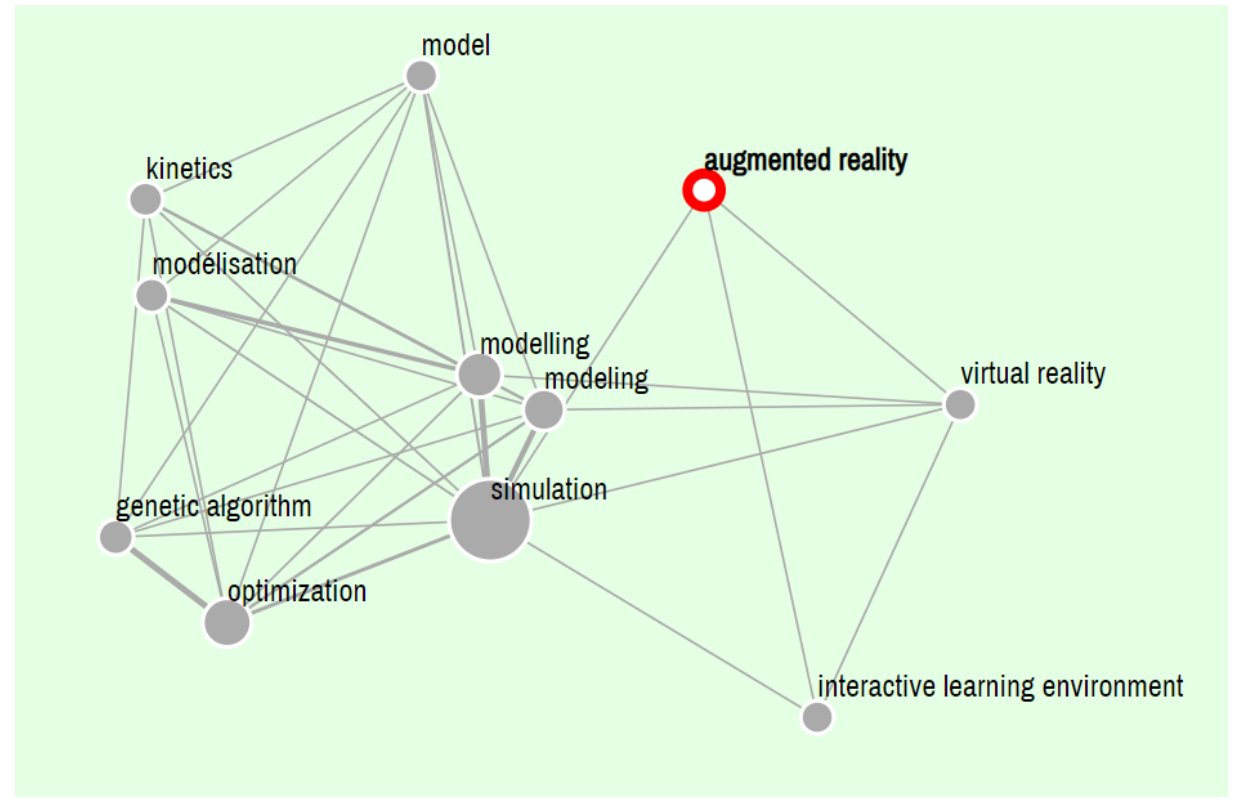

Figure 1. 'Augmented Reality' in B-Link

Producing ideas is challenging, while combinational creativity is considered one of the easiest approach for people. Combinational creativity involves producing new ideas through exploring unfamiliar associations between familiar ideas (Boden, 2004, 2009). Han et al. (2018a) have shown a computational tool, 'the Combinator', for supporting creative idea generation by simulating aspects of human cognition in producing combinational creative ideas. The tool can generate combinational stimuli in text- and image-forms through combining unrelated ideas. The ideas used for combination are from a customised database containing up-to-date product design knowledge and information, which was created through using web crawling, simple natural language processing, and an existing common-sense knowledge base. Thus, the tool could provide designers with creative combinational prompts involving up-to-date design information to support concept generation. For instance, many combinational ideas on 'navigation' could be produced by the Combinator, such as 'navigation 
history', 'navigation running, 'navigation watch', 'navigation car' and 'navigation shoe'. Examples of combinational prompts produced by the tool are shown in Figure 2.

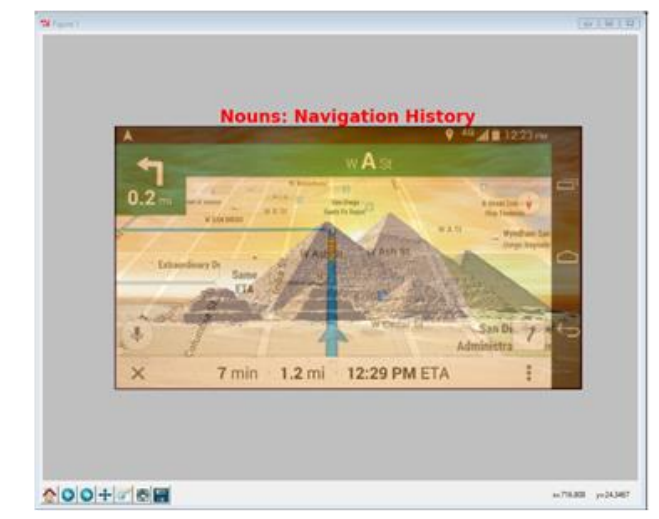

Navigation History

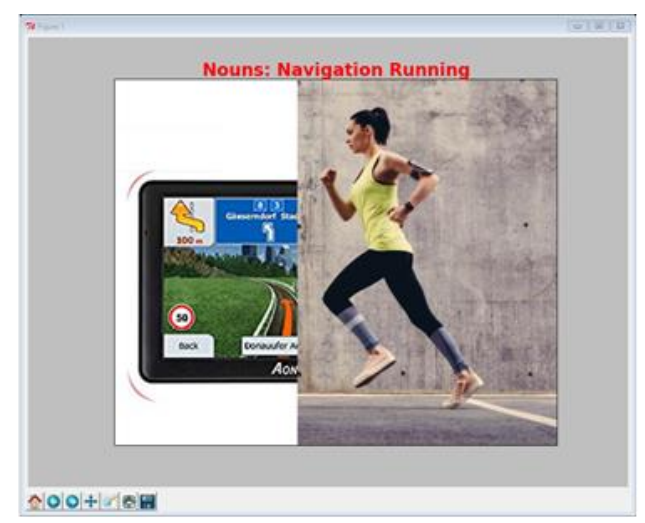

Navigation Running

Figure 2. Examples of combinational ideas about 'navigation' produced by the Combinator

The combinational prompts produced could be used to stimulate designers to generate new ideas about designing a new navigation application using augmented reality. As B-Link has demonstrated that 'augmented reality' is associated with 'interactive learning environment', 'modelling' and 'simulation', which has provided useful knowledge of 'augmented reality'. It has indicated that the best approaches to adopt 'augmented reality' to meet people's needs are to provide an interactive learning environment, simulation, modelling, and so on. Based on the generated combinational ideas and the provided knowledge of augmented reality, new concepts on navigation application using augmented reality could be produced, such as the ones shown in Figure 3.

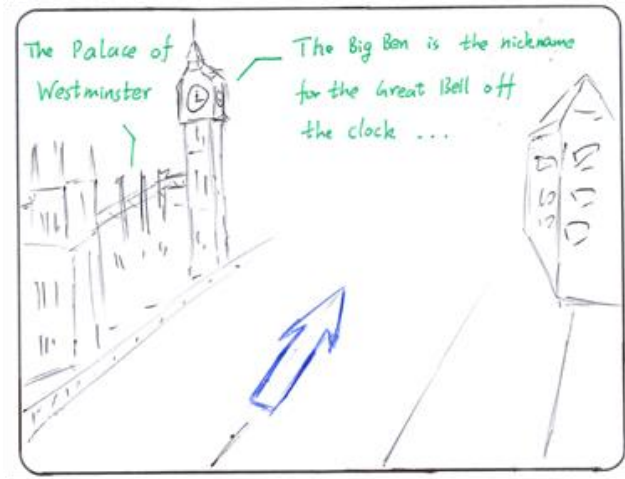

AR History Navigation Application

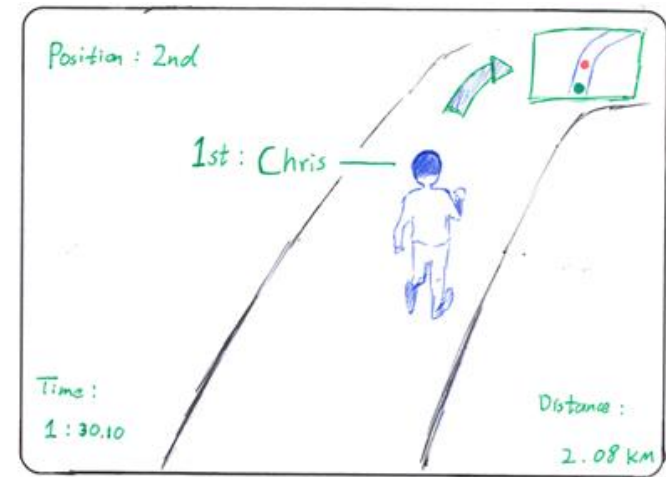

AR Running Navigation Application

$*$ Please note that the blue and green drawings indicate augmented reality, while the black drawings represents the reality.

Figure 3. Concepts generated based on the computational concept generation method

The AR history navigation application is a navigation system that provides users with additional interactive history information on buildings, sculptures, and so on. It helps the users learn the history of surrounding objects through offering an interactive learning environment using augmented reality technology during navigation. The AR running navigation application is a running system for smart glasses, which creates various running scenarios by modelling virtual rival runners and surroundings using augmented reality. For instance, it could train users in running by simulating a race competition.

\subsection{A computational concept evaluation method}

Concept generation is followed by concept evaluation. Using computational approaches could replace the level of expertise required, but few computational tools exist. In this section the authors propose a simple computational concept evaluation method to be implemented into the combinational-based computational concept generation method proposed. 
The distances between the ideas used for generating combinational ideas play a vital role in determining the creativity of the ideas produced. Several studies have explored how the distances between ideas or inspirational stimuli, such as analogies, relates to design idea outputs. Lopez et al. (2011) suggest distant analogies have greater potentials to produce more creative designs. Chan et al. (2011) show far analogies contribute to the novelty and variability of solutions. Han et al. (2018c) indicate that farrelated ideas could lead to more creative designs and are used more often than closely-related ones in practical designs. Goucher-Lambert and Cagan (2019) suggest that far inspirational stimuli could improve novelty, while near inspirational stimuli could improve feasibility and usefulness. However, Chan et al. (2015) indicate that design ideas employing closer related sources are more creative than the ones using further sources.

The majority of studies suggest far-related ideas could inspire more creative design outcomes. A computational evaluation algorithm could be integrated within the computational concept generation method to guide the combination process by associating far-related ideas rather than closely-related ones. Computational semantic distance measurement tools, such as SEMILAR (a semantic similarity toolkit) (Rus et al., 2013), ConceptNet (Speer and Havasi, 2012), and TechNet (Sarica et al., 2020) could be employed to identify the distances between ideas. Using the combinational ideas on navigation applications generated previously, the idea distances measured by ConceptNet and TechNet are shown in Table 1.

Table 1. Examples of idea distances measurement using ConceptNet and TechNet

\begin{tabular}{|ccc|}
\hline Combinational Ideas & ConceptNet [-1, 1] & TechNet [0, 1] \\
\hline Navigation History & 0.081 & 0.381 \\
\hline Navigation Running & -0.005 & 0.244 \\
\hline Navigation Watch & 0.011 & 0.307 \\
\hline Navigation Car & 0.154 & 0.237 \\
\hline Navigation Shoe & 0.023 & 0.115 \\
\hline
\end{tabular}

The distance value or relatedness value ranges from -1 to 1 in ConceptNet and 0 to 1 in TechNet, where a higher value indicates a closer distance. The ConceptNet and TechNet have shown different measurement results. The ConceptNet measurement results show that 'navigation running' is the combinational idea that has the largest distance, while 'navigation car' is the nearest. The TechNet results show that 'navigation history' and 'navigation shoe' are the nearest and farthest related idea, respectively. ConceptNet indicates 'navigation running' is the most creative combinational idea, while TechNet suggests 'navigation shoe'. Thereby, these ideas could be used to support designers produce creative design concepts. The computational concept evaluation approach employing idea distances could be implemented to guide the combinational-based concept generation method, such as, generating far-related combinational ideas rather than closely related ones. It allows concept generation for and with evaluation. However, different computational distance measurement tools use different algorithms and databases, and thereby leading to different results.

\subsection{The data-driven approach and discussions}

The sections above have presented a computational concept generation method and a computational concept evaluation method. Based on the two methods, a data-driven computational concept generation and evaluation approach is proposed, as shown in Figure 4.

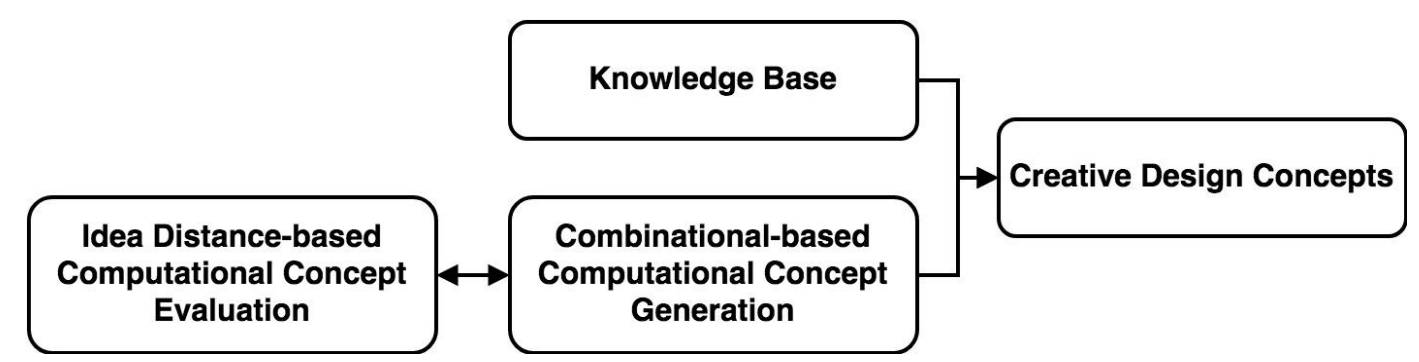

Figure 4. The data-driven computational concept generation and evaluation approach 
The data-driven computational concept generation and evaluation approach starts with providing designers with a knowledge base, such as the B-Link, to support the understanding of the relevant knowledge, such as emerging technologies, in the design context. After that, combinational-based computational techniques or tools, such as the Combinator, are used to produce combinational ideas or inspirational stimuli. Simultaneously, the ideas produced are evaluated computationally utilising idea distances. This could be conducted by using semantic distance measurement tools, such as ConceptNet. The evaluated combinational creative ideas are then produced in text- and image-forms. The computationally produced ideas together with the provided knowledge are then employed by designers to generate creative design concepts incorporating emerging technologies.

An example showing how the data-driven approach can be applied to design a navigation application using augmented reality, is presented in Figure 5. A knowledge base on 'augmented reality' is provided by B-Link to support the understanding of this new technology. For instance, the knowledge base shows 'augmented reality' is associated with 'interactive learning environment', 'modelling', and 'simulation', indicating how 'augmented reality' could be used. Many ideas, such as 'navigation history', 'navigation car' and 'navigation running' are produced by the Combinator. These combinational ideas are evaluated simultaneously according to their idea distances using ConceptNet. The computationally measured distance between 'navigation' and 'running' by ConceptNet is larger than the other ideas generated. This indicates 'navigation running' might be a more creative output idea. Thus, combinational prompts of 'navigation running' in both text- and image-forms and the 'augmented reality' knowledge base are employed by designers for supporting the generation of creative design concepts. Creative concepts incorporating the new technology 'augmented reality', such as the AR running navigation application for smart glasses, are then produced. The AR running navigation application is a running system employing augmented reality to model and simulate various running scenarios. This creative concept has demonstrated how the proposed approach could be used to incorporate emerging technologies in new products. Other knowledge bases, combinational algorithms, and concept evaluation methods could be used, but might lead to outcomes that are different from the provided example.

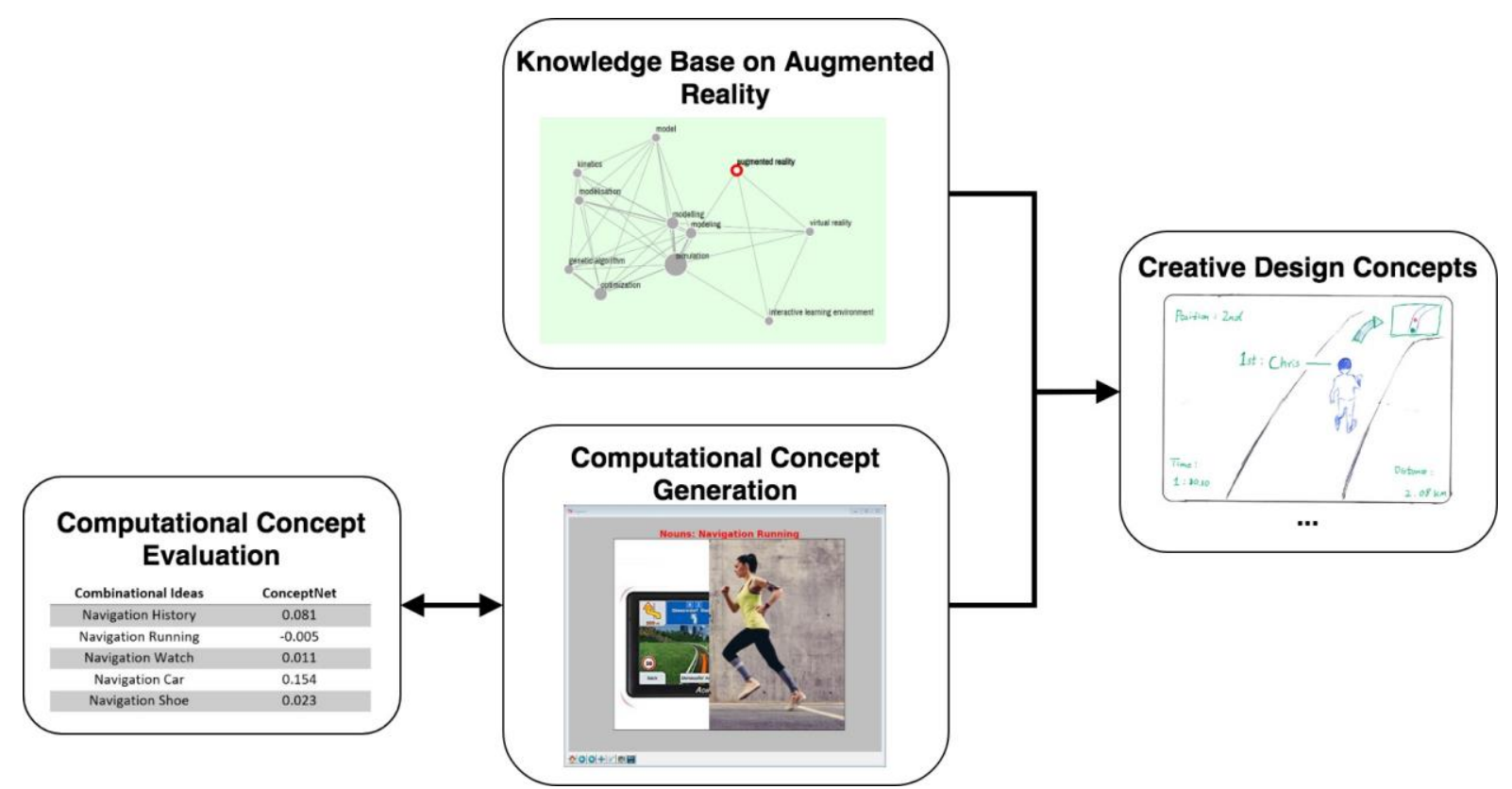

Figure 5. An example of using the data-driven approach to design a navigation application using augmented reality

As illustrated above, the data-driven computational concept generation and evaluation approach proposed in this paper could support designers to generate creative design concepts incorporating emerging technologies. However, further explorations of the knowledge base, computational concept generation and evaluation methods are needed to fully realize the approach. 
The current knowledge base, B-Link, is used to provide designers with the information required to understand emerging technologies. Other knowledge bases, such as TechNet and InnoGPS, could also be used as alternatives, which might provide designers with required information extracted from other sources, for example, patent database. The use of different knowledge bases provides designers with understandings of emerging technologies from different perspectives, which could affect the concept generated. Although the use of the knowledge base has significantly reduced designers' time on gathering information, the knowledge base needs to provide further explanation on the terms included.

The computational concept generation method, the Combinator, employed in the approach produces ideas in a random manner. Although randomness is an essential factor of creativity (Carruthers, 2011), He et al. (2019) has indicated that computer-based random combinations often lead to meaningless outcomes through an empirical study. Controlled computer-based combinations as well as human combinations have higher probabilities in producing novel and feasible outcomes (He et al., 2019). Therefore, there is still a need to explore how computational approaches guide the idea combination processes. In other words, there is a need to study how appropriate id eas for producing computational creative outputs should be selected. Alternatively, we could take the advantage of other existing knowledge base, such as the InnoGPS system (Luo et al., 2019). The system provides quantitative and visual guidance to concept combinations by informing the knowledge distances, which indicate the novelty and feasibility of the combinations. The indication is based on prior human experiments (Luo et al., 2018) as well as big data experiments (Alstott et al., 2017).

With regards to using idea distances for concept evaluation, other semantic measurement tools, such as SEMILAR and TechNet, as well as several existing computational idea evaluation methods such as the InnoGPS and the machine learning-based concept evaluation method proposed by Camburn et al. (2019; 2020), which have employed the use of semantic distances, could be used. However, further research is needed to explore the 'definition' of far-related and closely-related ideas in computational measurements. This could support the retrieval of far-related ideas for producing combinational ideas using computational techniques. However, far-related combinational ideas might cause potential difficulties in comprehension, especially to novice designers. It is much easier for novices to perceive a closely-related combinational idea than a far-related one, as novices are more likely to establish surface similarities between ideas (Ozkan and Dogan, 2013). In addition, some words have different meanings in various context, and thereby the distance between ideas changes according to where the word is situated. The distance value produced by many computational semantic measurement tools, such as ConceptNet and TechNet, do not reflect the polysemy of words. Thereby, further research on semantic distance measurement are needed, such as investigating whether the polysemy of words affect the measurement results, and new algorithms to measure semantic distances involving aspects of polysemy.

\section{Conclusion}

With increasingly emerging technologies, incorporating technologies into new products has become one of the main challenges in producing creative design concepts. In this paper, the authors proposed a data-driven design concept generation and evaluation approach to support designers, especially novices, in conceptual design to incorporate emerging technologies. The example provided in this paper has shown how the proposed approach could support designers in conceptual design. Potential further explorations on the knowledge base, computational concept generation and evaluation have been revealed to fully realize the approach. Besides, exiting knowledge databases, computational concept generation and evaluation tools, such as the TechNet and InnoGPS, will be employed in the proposed approach for comparison and yield additional insights. The proposed approach offers new insights on computational concept generation for and with evaluation. The new approach could support the creation of more efficient and capable products more regularly and faster than today. This has indicated potential future research directions in computational design support tools. Instead of focusing on either generation or evaluation activities, future design support tools should integrate both activities to enhance the efficiency and effectiveness of the tools. 


\section{References}

Alstott, J. et al. (2017), “Inventors' Explorations Across Technology Domains”, Design Science, Vol. 3, p. e20. https://doi.org/10.1017/dsj.2017.21

Bertola, P. and Teixeira, J.C. (2003), "Design as a knowledge agent: How design as a knowledge process is embedded into organizations to foster innovation”, Design Studies, Vol. 24 No. 2, pp. 181-194. https://doi.org/10.1016/ S0142-694X(02)00036-4

Boden, M.A. (2004), The creative mind: Myths and mechanisms, 2 ed., Routledge, London, UK.

Boden, M.A. (2009), "Computer models of creativity", AI Magazine, Vol. 30 No. 3, pp. 23-34. https://doi.org/ 10.1609/aimag.v30i3.2254

Brown, D.C. (2015), "Computational Design Creativity Evaluation”, in Design Computing and Cognition '14, Cham, Springer International Publishing. pp. 207-224.

Camburn, B. et al. (2020), "Machine Learning Based Design Concept Evaluation", Journal of Mechanical Design, In Press. https://doi.org/10.1115/1.4045126

Camburn, B. et al. (2019), "Evaluating Crowdsourced Design Concepts With Machine Learning", In Proceedings of ASME IDETC-CIE 2019. https://doi.org/10.1115/DETC2019-97285

Carruthers, P. (2011), "Creative action in mind", Philosophical Psychology, Vol. 24 No. 4, pp. 437-461.

Cash, P. and Štorga, M. (2015), "Multifaceted assessment of ideation: using networks to link ideation and design activity", Journal of Engineering Design, Vol. 26, pp. 391-415. https://doi.org/10.1080/09544828.2015. 1070813

Chakrabarti, A. and Shu, L.H. (2010), "Biologically inspired design”, Artificial Intelligence for Engineering Design, Analysis and Manufacturing, Vol. 24 No. 4, pp. 453-454. https://doi.org/10.1017/S08900604100 00326

Chan, J., Dow, S.P. and Schunn, C.D. (2015), "Do the best design ideas (really) come from conceptually distant sources of inspiration?”, Design Studies, Vol. 36 No. Supplement C, pp. 31-58. https://doi.org/10.1016/j. destud.2014.08.001

Chan, J. et al. (2011), "On the Benefits and Pitfalls of Analogies for Innovative Design: Ideation Performance Based on Analogical Distance, Commonness, and Modality of Examples", Journal of Mechanical Design, Vol. 133 No. 8, pp. 081004-081004-11. https://doi.org/10.1115/1.4004396

Chen, L. et al. (2019), “An artificial intelligence based data-driven approach for design ideation”, Journal of Visual Communication and Image Representation, Vol. 61, pp. 10-22. https://doi.org/10.1016/j.jvcir. 2019.02.009

Childs, P.R.N. (2018), Mechanical design engineering handbook, 2nd ed, Butterworth-Heinemann, Oxford, UK.

Eling, K., Griffin, A. and Langerak, F. (2014), "Using Intuition in Fuzzy Front-End Decision-Making: A Conceptual Framework", Journal of Product Innovation Management, Vol. 31 No. 5, pp. 956-972. https://doi.org/10.1111/jpim.12136

$\mathrm{Fu}$, K. et al. (2014), "Bio-Inspired Design: An Overview Investigating Open Questions From the Broader Field of Design-by-Analogy", Journal of Mechanical Design, Vol. 136 No. 11, pp. 111102-111102-18. https://doi.org/10.1115/1.4028289

Goucher-Lambert, K. and Cagan, J. (2019), "Crowdsourcing inspiration: Using crowd generated inspirational stimuli to support designer ideation”, Design Studies, Vol. 61, pp. 1-29. https://doi.org/10.1016/j.destud. 2019.01.001

Han, J., Forbes, H. and Schaefer, D. (2019), “An Exploration of the Relations between Functionality, Aesthetics and Creativity in Design", Proceedings of the Design Society: International Conference on Engineering Design, Vol. 1 No. 1, pp. 259-268. https://doi.org/10.1017/dsi.2019.29

Han, J. et al. (2018a), "The Combinator - a computer-based tool for creative idea generation based on a simulation approach", Design Science, Vol. 4, p. e11. https://doi.org/10.1017/dsj.2018.7

Han, J. et al. (2018b), "A computational tool for creative idea generation based on analogical reasoning and ontology”, Artificial Intelligence for Engineering Design, Analysis and Manufacturing, Vol. 32 No. 4, pp. 462-477. https://doi.org/10.1017/S0890060418000082

Han, J. et al. (2018c), "The conceptual distances between ideas in combinational creativity", in DS92: Proceedings of the DESIGN 2018 15th International Design Conference, pp. 1857-1866. https://doi.org/ 10.21278/idc.2018.0264

Hao, J., Zhao, Q. and Yan, Y. (2017), “A function-based computational method for design concept evaluation", Advanced Engineering Informatics, Vol. 32, pp. 237-247. https://doi.org/10.1016/j.aei.2017.03.002

He, Y. et al. (2019), "Mining and Representing the Concept Space of Existing Ideas for Directed Ideation", Journal of Mechanical Design, Vol. 141, p. 121101. https://doi.org/10.1115/1.4044399

Helms, M., Vattam, S.S. and Goel, A.K. (2009), "Biologically inspired design: process and products", Design Studies, Vol. 30 No. 5, pp. 606-622. https://doi.org/10.1016/j.destud.2009.04.003 
Howard, T.J., Culley, S. and Dekoninck, E.A. (2011), "Reuse of ideas and concepts for creative stimuli in engineering design", Journal of Engineering Design, Vol. 22 No. 8, pp. 565-581. https://doi.org/10.1080/ 09544821003598573

Hsu, W. and Liu, B. (2000), “Conceptual design: issues and challenges”, Computer-Aided Design, Vol. 32 No. 14, pp. 849-850. https://doi.org/10.1016/S0010-4485(00)00074-9

Jonson, B. (2005), "Design ideation: the conceptual sketch in the digital age", Design Studies, Vol. 26 No. 6 , pp. 613-624. https://doi.org/10.1016/j.destud.2005.03.001

Luo J., Sarica S. and Wood K. (2019), "Computer-Aided Ideation Using InnoGPS", in Proceedings of ASME IDETC-CIE 2019. https://doi.org/10.1115/DETC2019-97587

Luo, J. et al. (2018), "Design Opportunity Conception Using Technology Space Map", Artificial Intelligence for Engineering Design, Analysis and Manufacturing, Vol. 32 No. 4, pp. 449-461. https://doi.org/ 10.1017/S0890060418000094

Lopez, R., Linsey, J.S. and Smith, S.M. (2011), "Characterizing the Effect of Domain Distance in Design-byAnalogy", in Proceedings of the ASME 2011 International Design Engineering Technical Conferences and Computers and Information in Engineering Conference, pp. 141-151. https://doi.org/10.1115/DETC201148428

Oman, S.K. et al. (2013), "A comparison of creativity and innovation metrics and sample validation through inclass design projects", Research in Engineering Design, Vol. 24 No. 1, pp. 65-92. https://doi.org/ 10.1007/s00163-012-0138-9

Ozkan, O. and Dogan, F. (2013), “Cognitive strategies of analogical reasoning in design: Differences between expert and novice designers", Design Studies, Vol. 34 No. 2, pp. 161-192. https://doi.org/10.1016/j.destud. 2012.11.006

Rus, V. et al. (2013), "Semilar: The semantic similarity toolkit", in Proceedings of the 51st Annual Meeting of the Association for Computational Linguistics: System Demonstrations, pp. 163-168.

Sarica, S., Luo, J. and Wood, K.L. (2020), “TechNet: Technology Semantic Network", Expert Systems with Applications, Vol. 142, p. 112995.

Sarica, S., Song, B., Luo, J. (2019), “Technology Knowledge Graph for Design Exploration: Application to Designing the Future of Flying Cars", in Proceedings of ASME IDETC-CIE 2019. https://doi.org/10.1115/ DETC2019-97605

Sarkar, P. and Chakrabarti, A. (2011), “Assessing design creativity”, Design Studies, Vol. 32 No. 4, pp. 348-383.

Shah, J.J. et al. (2003), "Empirical Studies of Design Ideation: Alignment of Design Experiments With Lab Experiments", in Proceedings of the ASME 2003 International Design Engineering Technical Conferences and Computers and Information in Engineering Conference, pp. 847-856. https://doi.org/10.1115/ DETC2003/DTM-48679

Shi, F. et al. (2017), “A Data-Driven Text Mining and Semantic Network Analysis for Design Information Retrieval”, Journal of Mechanical Design, Vol. 139 No. 11, pp. 111402-111402-14. https://doi.org/ $10.1115 / 1.4037649$

Speer, R. and Havasi, C. (2012), "Representing general relational knowledge in ConceptNet 5", in Proceedings of the Eight International Conference on Language Resources and Evaluation.

Ullman, D.G. (2010), The mechanical design process: Part 1, McGraw-Hill, New York, USA.

Yilmaz, S. et al. (2016), "Evidence-based design heuristics for idea generation", Design Studies, Vol. 46 No. Supplement C, pp. 95-124. https://doi.org/10.1016/j.destud.2016.05.001

Zhang, Z.-J. et al. (2017), "A quantitative approach to design alternative evaluation based on data-driven performance prediction”, Advanced Engineering Informatics, Vol. 32, pp. 52-65. https://doi.org/10.1016/ j.aei.2016.12.009 